\title{
3D foot joints angle description using projected lines on anatomical planes
}

\author{
Hossein Rouhani*1, Julien Favre ${ }^{1}$, Xavier Crevoisier ${ }^{2}$, Brigitte $\mathrm{M}$ Jolles ${ }^{2}$ and \\ Kamiar Aminian ${ }^{1}$
}

Address: ${ }^{1}$ Ecole Polytechnique Fédérale de Lausanne (EPFL-LMAM), Lausanne, Switzerland and ${ }^{2}$ Centre Hospitalier Universitaire Vaudois \& University of Lausanne, Lausanne, Switzerland

Email: Hossein Rouhani* - hossein.rouhani@epfl.ch

* Corresponding author

from Ist Congress of the International Foot \& Ankle Biomechanics (i-FAB) community

Bologna, Italy. 4-6 September 2008

Published: 26 September 2008

Journal of Foot and Ankle Research 2008, I (Suppl I):O3 I doi:I0.II86/I757-I |46-I-SI-O3 I

This abstract is available from: http://www.jfootankleres.com/content/I/SI/O3I

(c) 2008 Rouhani et al; licensee BioMed Central Ltd.

\section{Introduction}

An increasing trend for 3D kinematic analysis of multisegment foot has emerged both in clinical and biomechanical studies $[1,2]$. Nevertheless, existence of several joints among tiny bones of foot causes difficulties both for joint angle definition and their measurement. Unlike with the larger lower limbs bone, it is hardly possible to put three reflective markers over each foot bone. Subsequently, it is not practical to define local coordinate systems (LCS) for all foot segments bounding foot joints. This work proposed a simple method for foot joints angle description which is easy to interpret.

\section{Methods}

Since it is hardly possible to have LCS based on three rigid markers per foot segment, many of standard joint angle description methods (e.g. JCS) might not be suitable for many foot joints. Moreover, such conventional methods might not be efficient for some foot joint angles descriptions in clinical applications.

Recently, Simon et al [2] proposed a new foot joint angle description where each joint angle is defined by the rotation angle between two lines corresponding to two segments about a rotation axis on the third segment. The defined joint angle is equal with the angle between projections of two lines on the normal plane of the rotation axis. The results were repeatable and reliable. Nevertheless, a)

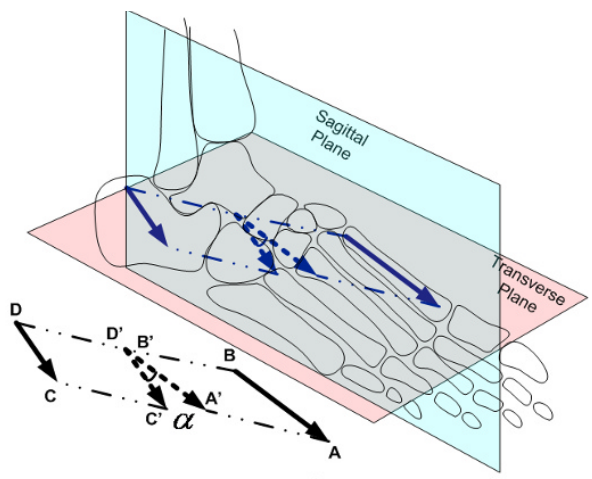

b)

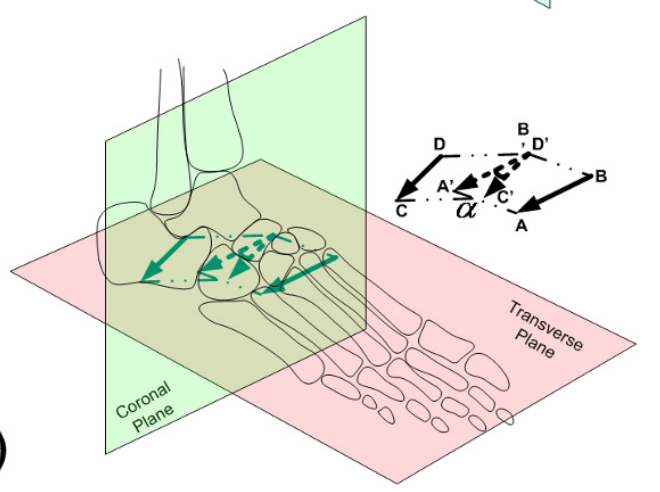

Figure I

Definition of calcaneus-metatarsals joint angles: a) Lines defined for sagittal projection b) Lines defined for coronal projection. 
there was a need for defining one axis for each considered joint which complicates the 3D kinematic model of foot. We proposed to define all foot joint angles by projection on three common anatomical planes.

Sagittal, coronal and transverse planes of whole foot were defined based on [3]. For each considered foot joint, one line per each of two segments bounding the joint was defined between captured anatomical landmarks (AL). As an example, Figure 1 depicts this method for the case of angle calculation for the considered joint between calcaneus and metatarsals.

\section{Results}

The proposed method was applied for foot kinematic analysis of 6 subjects with 21 tiny markers on ALs of foot. The $3 \mathrm{D}$ joint angle patterns and ranges were comparable to those of the other methods [1]. For similar angles, repeatability and sensitivity to measurement errors were showed to be in the same range as other angle representation methods.

\section{Conclusion}

A method to describe 3D angles of foot joints was suggested with an easy clinical interpretation. Without use of LCS, the method had also the similar performance as other conventions. Yet, the movement of foot's anatomical planes can induce error which can be avoided by definition of rotation axis on the same joint segments and not others. The suitability of method will be soon assessed in clinical applications.

\section{References}

I. Leardini A, et al.: Gait \& Posture 2007, 25:453-462.

2. Simon J, et al.: Gait \& Posture 2006, 23:4 I I-424.

3. Cappozzo A, et al.: Clinical Biomechanics 1995, I0(4): 17।- 178. 